\title{
Constipation and reversible urinary tract abnormalities
}

\author{
R Dohil, E Roberts, K Verrier Jones, H R Jenkins
}

\begin{abstract}
Urinary tract anomalies were prospectively investigated with ultrasound in 29 children with functional constipation. These children were compared before and after treatment with 451 age matched healthy controls without constipation. The bladder residue and upper renal tract dilatation after micturition were significantly increased in the group with constipation and improved after treatment. (Arch Dis Child 1994; 70: 56-57)
\end{abstract}

Functional constipation, defined as constipation without evidence of an underlying abnormality, is a common and underdiagnosed paediatric problem. A number of reports have linked constipation with urinary tract problems, including urinary tract infection, enuresis, vesicoureteric reflux, and upper renal tract dilatation, ${ }^{1-4}$ though a mechanism for the increased incidence of these abnormalities has not been clearly defined.

With the aim of investigating this relation we undertook a prospective controlled study to evaluate the urinary tract in a series of children with constipation using ultrasound. We observed the effects of treatment and compared the children with controls. Ethical approval was obtained from the local research ethics committee.

\section{Patients and methods}

Twenty nine consecutive children with functional constipation, aged $2-14$ years (10 boys, 19 girls), were identified during a three month period in the gastroenterology (15) and renal (14) clinics after a history of infrequent bowel action (less than three actions each week). All described additional features such as large, hard stools, straining, and soiling. All had passed meconium at the normal time and none had clinical evidence of neurological abnormalities nor a history of anal fissure nor Hirschsprung's disease and, in every child, the diagnosis of constipation was confirmed on a plain abdominal radiograph which was used to exclude spinal dysraphism.

After confirmation of constipation, the nature of the study was explained and consent obtained. Initial bowel clearout was induced by sodium picosulphate by mouth and three children required a phosphate enema. In addition to the usual dietary advice and modification of behaviour, parents were instructed to give lactulose and Senokot on a sliding scale to achieve at least five soft bowel actions each week. Control data were obtained by ultrasound from 451 healthy children aged 2-14 years and studies after micturition were carried out on 111 of the controls.

The children were given a large drink 30-45 minutes before the ultrasound study, which was undertaken initially with a full bladder and then repeated after emptying. Bladder, kidneys, and ureters were evaluated using a standard technique by one ultrasonographer (ER) using a Siemens Sonoline SX portable ultrasound scanner and $5 \mathrm{MHz}$ transducer. Bladder volume was estimated from three dimensional measurements and the diameters of the pelvicalyceal systems and upper ureters were measured and any renal anomalies noted. The measurements were repeated after micturition. Ultrasound studies were carried out at the initial assessment and repeated every two to four weeks until a regular bowel habit had been established.

Results were compared before and after treatment and with controls using the Wilcoxon matched pair and signed rank tests, and the Mann-Whitney and Fisher's exact tests.

\section{Results}

Urinary tract infection was confirmed on midstream urine culture by finding $>10^{5}$ colony forming units $/ \mathrm{ml}$ on a single urine sample in 12 of the 14 children with urinary symptoms. A residue after micturition was detected in 19 of 29 children $(66 \%)$ in the study group at initial assessment and six of 29 children $(21 \%)$ at

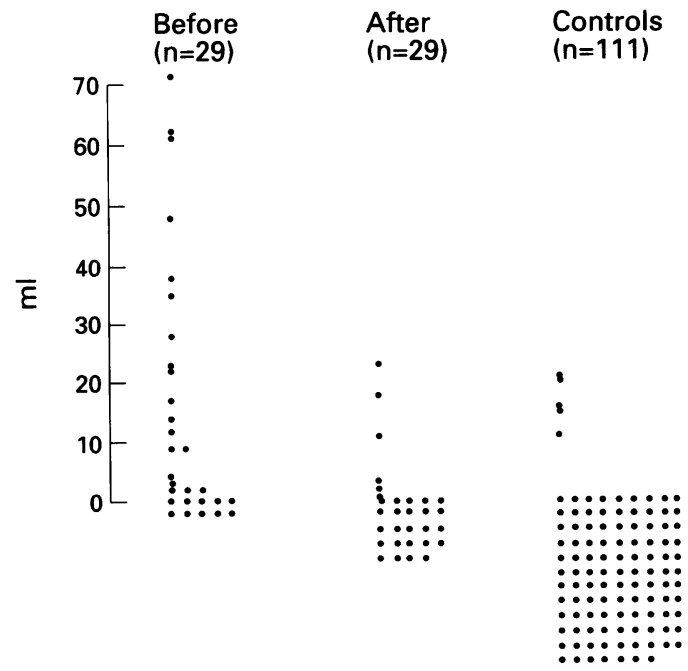

Ultrasound assessment of residue after micturition in 29 children with constipation before and after treatment compared with 111 controls. 
No (\%) of children with pelvicalyceal diameter $>7 \mathrm{~mm}$ : children with constipation before and after treatment and controls

\begin{tabular}{lcc}
\hline Subjects & Right kidney & Left kidney \\
\hline Children with constipation & & \\
Before treatment & $7 / 29(24)$ & $4 / 29(14)$ \\
After treatment & $2 / 29(7)$ & $2 / 29(7)$ \\
Controls & $15 / 451(3)$ & $11 / 451(3)$ \\
& &
\end{tabular}

Before treatment $v$ controls $\mathrm{p}<0 \cdot 05$.

final assessment after treatment, compared with five of 111 controls (5\%) (figure). The residue after micturition was significantly more common in children with constipation before treatment compared with controls $(p<0.001$, Mann-Whitney test) and was significantly reduced by treatment $(p<0.001$, Wilcoxon matched pairs signed rank test). The median reduction in the residue after micturition was $10.5 \mathrm{ml}(95 \%$ confidence interval 2 to $19 \mathrm{ml})$. Even after treatment, however, the study group showed a residue after micturition more often than the controls $(p<0 \cdot 013$, Fisher's exact test).

Pelvicalyceal dilatation was present in 11 of $29(38 \%)$ constipated children before treatment (table) compared with 26 of $451(5 \cdot 8 \%)$ controls $(p<0.05$ using Fisher's exact test) and, though there was a reduction in the number of constipated children with dilated kidneys $(>7 \mathrm{~mm})$ after treatment from seven to two on the right kidney and from four to two on the left kidney, these improvements did not reach statistical significance. Three children with nocturnal enuresis had a significant residue after micturition and, after treatment, this residue had been abolished and the symptoms resolved. Two children had had recent recurrent episodes of acute retention, but had no further attacks after treatment.

Four children (two from the renal clinic and two from the gastroenterology clinic) showed ultrasound evidence of renal scarring and, in two patients, this was associated with upper tract dilatation, though neither of these children was shown to have vesicoureteric reflux at micturating cystourethography.

\section{Discussion}

Constipation is a common paediatric problem which remains underdiagnosed and poorly treated. Although the associated gastrointestinal and psychological symptoms are well recognised, the effects on the urinary tract are not so widely appreciated, with the result that constipation may not be suspected immediately when a child presents with urinary symptoms and, conversely, when constipation and soiling are a major source of anxiety to a family, urinary symptoms and difficulties are easily overlooked.

The close anatomical proximity of the bladder and urethra to the rectum, and the similar spinal innervation (S4-S4) of the urethral and anal sphincters, make it likely that abnormalities within one system may affect the other. Indeed, Shopfner has shown that a faecally impacted rectum will cause irregularities of the posterior bladder wall. ${ }^{3}$

Although this study was not designed to determine the incidence of constipation in children with urinary tract infection, we have shown that urinary tract abnormalities often occur in children with functional constipation and these abnormalities appear to be reversible after adequate treatment. It is interesting to note that the children presenting with constipation were usually boys and that the children presenting with urinary symptoms were usually girls. Lindberg et al showed that, among schoolgirls with bacteriuria, those with a residue after micturition were more prone to recurrent urinary tract infections. ${ }^{5} \mathrm{He}$ did not look for constipation within his study group, however. O'Regan and Yasbeck showed the presence of inappropriate detrusor muscle contraction in children with constipation, ${ }^{4}$ and it has been shown that incomplete bladder emptying provides a reservoir for bacteria and may predispose to urinary tract infection. ${ }^{6}$

Upper tract dilatation was reported by Herbetko and Hyde, who showed that, of seven children with functional constipation and upper renal tract dilatation, only one had vesicoureteric reflux on micturating cystogram..$^{2}$ In these children it is therefore likely that the pelvicalyceal system dilatation had arisen from the mechanical compression of the urinary tract by the distended rectum, which may occur at the level of the urethra, bladder, or the vesicoureteric junction.

Our study has shown that potentially serious renal tract abnormalities are relatively common in children with functional constipation and these are, to a large extent, reversible. Therefore our findings provide additional reasons for the prompt and effective treatment of constipation and we recommend that constipation should be carefully excluded in children presenting with urinary tract infections, enuresis, and urinary retention.

The authors thank Miss Leah Gallivan and Mrs Christa George for preparation of the manuscript, the Laura Ashley Foundation for financial support, and Dr Robert Newcombe for his assistfor financial support, and Dr Ro
ance the statistical analysis.

1 O'Regan S, Yasbeck S, Schick E. Constipation, bladder instability, urinary tract infection syndrome. Clin Nephrol 1985; 23: 152-4.

2 Herbetko J, Hyde I. Urinary tract dilatation in constipated children. Br ₹ Radiol 1990; 63: 855-7.

3 Shopfner CE. Urinary tract pathology associated with constipation. Radiology 1968; 90: 865-77.

4 O'Regan S, Yasbeck S. Constipation: a cause of enuresis, urinary tract infection and vesico-ureteric reflux in childurinary tract infection and vesico-urete

5 Lindberg U, Bjure J, Haugstredt J, Jodal U. Asymptomatic bacteruria in schoolgirls. Acta Paediatr Scand 1975; 64: 437-40

6 O'Grady F, Gauci DL, Watson BW, Hammond B. In vitro models simulating conditions of bacterial growth in the urinary tract. In: O'Grady F, Brunfitt W, eds. Urinary tract infection. London: Oxford University Press, 1968: 80. 